(74.5 \pm 7 years, $46.4 \%$ male) without dementia collected salivary cortisol after awakening and late in the evening. After 4 years, 852 persons received follow-up. At baseline and follow-up, global cognitive functioning, verbal memory performance, and processing speed were assessed using the MMSE; the 15 Word Learning Test (verbal learning during 3 trials, delayed recall, and memory retention (\%)); and a digit-coding task (score range 0-50). Using linear mixed models adjusted for age, gender, education, smoking, alcohol use, BMI, hypertension, diabetes, vascular disease, and depressive symptoms, the cross-sectional and longitudinal associations of awakening and evening cortisol and diurnal variability (per $\mathrm{nmol} / \mathrm{l}$ increase) with cognitive outcomes were estimated. Results: Crosssectionally, higher evening cortisol levels were associated with poorer delayed recall $(\mathrm{B}=-0.05 ; 95 \% \mathrm{CI}-0.09$ to -0.01$)$ and poorer memory retention ( $\mathrm{B}=-0.42 ; 95 \% \mathrm{CI}-0.70$ to -0.14$)$, but not with verbal learning. High diurnal variability of cortisol was related to better scores on verbal learning $(\mathrm{B}=0.13 ; 95 \% \mathrm{CI} 0.02$ to 0.24$)$; better delayed recall $(\mathrm{B}=0.08$; $95 \%$ CI 0.02 to 0.14$)$; and better memory retention $(\mathrm{B}=0.59 ; 95 \%$ CI 0.13 to 1.04). No associations were found between salivary cortisol and MMSE or processing speed. Also, awakening levels were not associated with any of the cognition measures. Although all cognitive scores declined over time, no longitudinal associations were observed between any of the cortisol measures and cognitive decline for any cognitive test. Conclusions: In this older non-demented population, higher levels of evening cortisol and low diurnal variability of cortisol are associated with poorer verbal memory performance, but not with memory decline or decline in other cognitive domains after 4 years of follow-up. Our findings do not support the hypothesis that excessive glucocorticoids increase the risk for Alzheimer's disease.

\section{P4-026 USE OF STATINS, LDL-C AND INCIDENCE OF COGNITIVE IMPAIRMENT OR DEMENTIA IN A SEVEN-YEAR COHORT STUDY OF OLDER MEXICAN-AMERICANS}

Mary N. Haan, Caryn Cramer, University of Michigan, Ann Arbor, MI, USA. Contact e-mail: mnhaan@umich.edu

Background: Evidence for an association between statins and dementia is mixed. This study aims to examine the association between statin use and incidence of dementia and cognitive impairment without dementia over 7 years of follow-up. Methods: The Sacramento Area Latino Study on Aging (SALSA- AG12975) is a population-based cohort study of 1,789 Mexican-Americans aged 60-101 done from 1998-2008. Participants had interviews and cognitive evaluations performed every 12 to 15 months. Those who fell below the 20th percentile on at least one of two cognitive tests (Modified Minimental State Exam or delayed word list recall) were evaluated clinically. Diagnosis of dementia or cognitive impairment without dementia (CIND) was finalized by an expert adjudication team. A total of 1674 participants without dementia/CIND at baseline were included in these analyses. Dementia/CIND were combined to increase statistical power. Statin use was verified at a participant's home visit by medicine cabinet inspection and classified using a CDC standardized system. LDL-C was obtained from a fasting blood sample. Cox proportional hazards models were used to evaluate the association between statin use over time and combined incidence of dementia/CIND. Results: Overall, 452 of 1674 participants $(27 \%)$ took statins at any time during the study. The most commonly used statins on average over time were: Atorvastatin (46\%), lovastatin (29\%), pravastatin (26\%), and simvastatin (22\%). Statin use was associated with a greater decline in LDL-C over the follow up period (-22 vs. $-7.8 \mathrm{ug} / \mathrm{dl}, \mathrm{p}<.0001)$. In all, 152 participants developed dementia/ CIND. Statin users experienced lower rates of dementia/CIND than nonusers $(\mathrm{HR}=0.52,95 \% \mathrm{CI}$ : $0.34,0.80)$ in proportional hazards regression models adjusted for education, smoking status, APOE $\epsilon 4$, and baseline stroke or diabetes. LDL-C was associated with a lower risk of dementia/ CIND (HR for interquartile range of $45 \mathrm{ug} / \mathrm{dl}$ : 0.70, $\mathrm{p}=0.003$ ); effect modification of this association by statin use was not significant $(\mathrm{p}=0.33)$. LDL-C adjustment reduced the statin-dementia association by $26 \%$. Con- clusions: Statins are linked to lower risks of cognitive impairment and lower LDL-C may be a partial mediator of this association. However, LDL-C is associated with cognitive impairment after adjustment for statin. Statins may influence neurodegeneration through non lipid pathways.

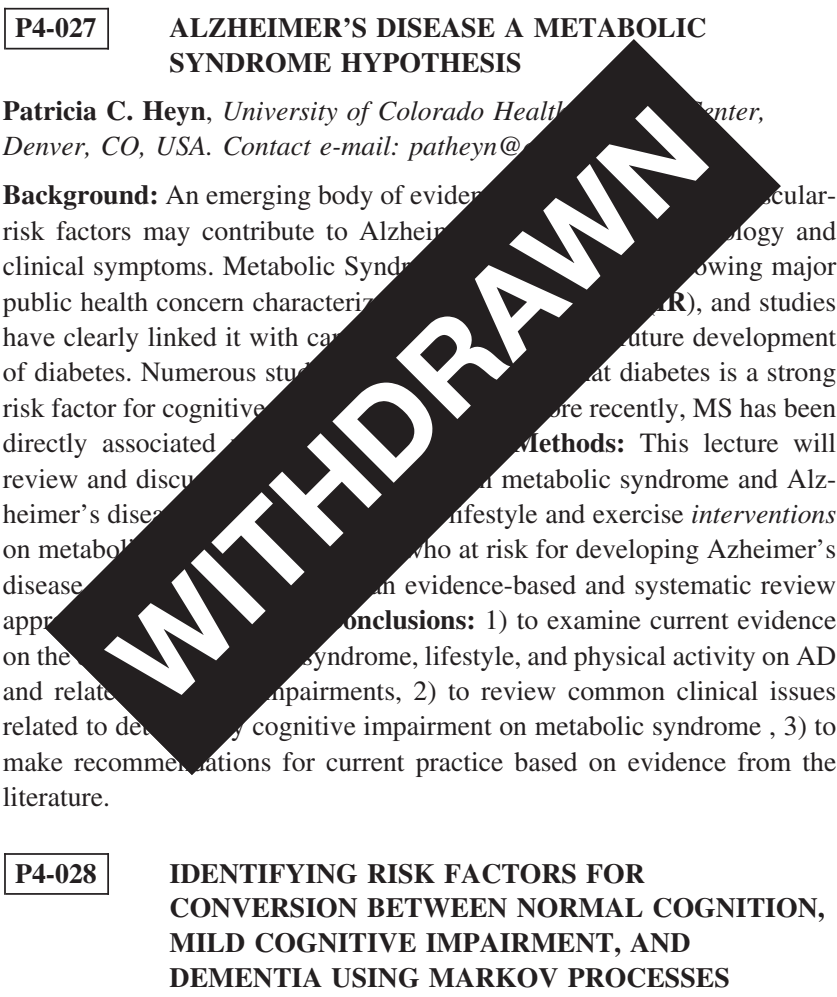

Rebecca Hubbard, University of Washington, Seattle, WA, USA. Contact e-mail: rebecca.hubbard@gmail.com

Background: Subjects with mild cognitive impairment (MCI) are at heightened risk of conversion to dementia. Using longitudinal information on changes in risk factors has the potential to substantially improve our ability to identify subjects at greatest risk of conversion. However, limited statistical methods are available for modeling multi-state disease processes in which the rate of transition between disease states is age-dependent and exact time of transition between states may be unknown, both of which are issues in studies of Alzheimer's disease. Methods: We have developed a new statistical model for estimating the effect of risk factors on conversion from normal cognition to MCI and dementia. This model makes use of a Markov process to characterize risk factors for transition between multiple disease states. We applied this model to the National Alzheimer's Coordinating Center's Uniform Data Set, a longitudinal study of subjects evaluated at one of the National Institute on Aging's Alzheimer's Disease Centers. We used our model to estimate risk of progression to amnestic MCI and dementia for 2724 subjects who had at least two observations. Risk factors considered in our model were years of education, history of dementia in a first degree relative, and MCI affecting additional domains beyond memory. Results: Our model indicated strong age-dependent trends in rates of conversion between all disease states. We additionally found that subjects with a history of dementia in a first degree relative were significantly more likely to transition from normal cognition to MCI (hazard ratio: 1.918, 95\% CI: $(1.02,3.60)$ ) after adjusting for education and multi-domain MCI. Conclusions: Our model confirms some previously identified risk factors for conversion to MCI and illustrates the way in which multi-state models can be used to identify risk factors for conversion. This type of statistical model is necessary to appropriately account for features of the Alzheimer's disease process including age-related changes in conversion rates and imprecisely observed times of transition between 